University of Nebraska - Lincoln

DigitalCommons@University of Nebraska - Lincoln

\title{
Effects of Xenobiotics and Phytotoxins on Reproduction in Food Animals
}

Kip E. Panter

USDA-Agricultural Research Service, Kip.panter@ars.usda.gov

Bryan L. Stegelmeier

USDA-Agricultural Research Service

Follow this and additional works at: https://digitalcommons.unl.edu/usdaarsfacpub

Part of the Agricultural Science Commons

Panter, Kip E. and Stegelmeier, Bryan L., "Effects of Xenobiotics and Phytotoxins on Reproduction in Food Animals" (2011). Publications from USDA-ARS / UNL Faculty. 870.

https://digitalcommons.unl.edu/usdaarsfacpub/870

This Article is brought to you for free and open access by the U.S. Department of Agriculture: Agricultural Research Service, Lincoln, Nebraska at DigitalCommons@University of Nebraska - Lincoln. It has been accepted for inclusion in Publications from USDA-ARS / UNL Faculty by an authorized administrator of DigitalCommons@University of Nebraska - Lincoln. 


\section{Effects of Xenobiotics and Phytotoxins on Reproduction in Food Animals}

Kip E. Panter, PhD*, Bryan L. Stegelmeier, DVM, PhD

\section{KEYWORDS}

- Phytotoxins • Plants • Xenobiotics • Reproduction • Animals

The influence of natural toxicants and anthropogenic compounds on reproduction in food animals is significant in its economic impact, and the subject requires more research and further experimental substantiation. Confounding factors such as stress, nutritional status, season of the year, animal species involved, genetic variability, disease conditions, management factors, and so forth exacerbate the difficulty of making an accurate diagnosis and thereby may impede progress to improve reproductive performance on an individual operation. The interaction between the reproductive system and xenobiotics (reproductive toxicology) is a relatively new area of study and a subject of increasing interest, especially in the area of environmental exposures and potential work place toxicants affecting human health and reproduction. ${ }^{1}$ Much of the experimental literature about this subject comes from rodent models designed to replicate human exposure; however, the extrapolation to food-producing animals is limited at best. The list of compounds in this article with known effects on reproductive function is extensive and represents most classes of chemicals in the environment; however, this list is not intended to be exhaustive.

Investigation of reproductive dysfunction, especially infertility, abortions, and teratogenesis, should center on a thorough examination of animal condition and health, management practices, and infectious agents while potential toxicants are sought. This method requires a systematic approach including individual animal and herd/ flock history, veterinary examination of individual animals, testing of blood, urine, feces, or tissues, gross and pathologic/histologic postmortem examination, and

The authors have nothing to declare.

Poisonous Plant Research Laboratory, USDA-Agricultural Research Service, 1150 East 1400

North, Logan, UT 84341, USA

* Corresponding author.

E-mail address: Kip.panter@ars.usda.gov 
toxicologic screening of samples of feed and or tissue. In livestock production systems, these investigations are often limited by economics, and the extent of the battery of tests must be determined in consultation between the animal producer, veterinarian, and diagnostician. Reproductive dysfunction includes all facets of reproduction, and when such dysfunction occurs failure to conceive, abortion, stillbirths, and anomalous fetuses may result.

Although the following discussion focuses on abnormal embryonic and fetal development (teratogenesis), many of the principles and methods outlined in this article may be used to investigate the other causes of reproductive dysfunction.

\section{DEVELOPMENTAL TOXICOLOGY (TERATOGENESIS)}

Although the exact molecular mechanism(s) is (are) unknown for many developmental anomalies, the production of an abnormal phenotype may be the result of a single (or multiple) defect(s) in the genotype, environmental insult, or animal-environmental interaction. This process results in tissues either failing to differentiate and develop or in incorrect tissue-tissue interactions. Subsequently, these failures impair normal development. Abnormal development occurs when a threshold of genetic and environmental insults is reached and the fetal compensatory mechanisms are overwhelmed; however, abnormal development is only part of the story of reproductive toxicology.

Six basic principles of teratology were originally described by Wilson ${ }^{2}$ in 1959 and further defined in 1977. These principles have withstood the test of time and are applicable for not only developmental toxicology but for other types of reproductive dysfunction.

1. Genetic susceptibility. Embryonic/fetal susceptibility to teratogens depends on the interaction of genotype and putative teratogen.

2. Time of exposure. Developmental stage of the embryo/fetus at exposure or insult often determines the type of defect. The embryo is often more sensitive than the fetus because this is the stage of organogenesis. However, there are numerous examples whereby the teratogen adversely affects fetal development at various stages of pregnancy, such as lupine-induced "crooked calf disease." 3

3. Pathogenesis of defective development. Teratogenic agents may act on cells or tissues by specific biochemical mechanisms to induce the abnormal development.

4. Definition of abnormal development. The final manifestation of abnormal development may include death with subsequent resorption or abortion, morphologic malformations, growth retardation, behavioral anomalies, or organ system dysfunction.

5. Chemical nature of the teratogen. The access and adverse influence to embryonic or fetal tissues or organs depend on the biologic, chemical, or physical nature of the teratogen.

6. Dosage. The amount of teratogen and size of dam influence the degree of insult from little or no effect to lethality. The effects and/or severity of the toxin or teratogen is dose dependent.

Many genetic, bacterial, and viral factors are responsible for certain malformations, and some of these may mimic defects induced by plant or chemical teratogens, thus confounding a diagnosis.

Causes of many human congenital malformations remain obscure, with estimates of $20 \%$ to $25 \%$ of developmental defects being attributable to genetic anomalies, $5 \%$ to identifiable toxicants, and the vast majority $(40 \%-60 \%)$ to unknown causes 
associated with gene-toxicant interactions. ${ }^{4,5}$ While Wilson's principles defining abnormal development have been validated consistently over time, the mechanisms responsible for xenobiotic-induced congenital defects remain elusive, and few studies have identified the pathways of the abnormalities. However, this area of research is rapidly progressing by using molecular tools superimposed on toxins with known effects. $^{6,7}$ Table 1 outlines teratogenic plants and Table 2 documents other xenobiotics with teratogenic effects.

\section{ABORTION-INDUCING TOXICANTS}

Certain natural toxins from plants, fungi, and man-made toxicants have been implicated or associated with abortion, embryonic death, or neonatal loss. In Tables 3-6 the toxicants of significance to animals are listed, accompanied by the chemical name of the toxicant (if known), the clinical effects, and species affected. Table 4 specifically lists pines, junipers, and other tree and shrub species that contain the abortifacient toxin isocupressic acid (ICA) or ICA derivatives associated with the so-called pine-needle abortion syndrome in cattle. ${ }^{10,11}$ ICA was identified as the primary abortifacient in cattle from Ponderosa pine, ${ }^{10}$ and since its discovery numerous tree and shrub species have been screened and found to contain ICA or related compounds (see Table 4). As toxin-induced abortions are relatively rare, more frequent causes should be excluded first. The differential diagnosis should also include bacterial or viral agents such as salmonellosis, brucellosis, leptospirosis, mycotic placentitis, bovine viral diarrhea (BVD), infectious bovine rhinotracheitis (IBR), and so forth, and nutritional factors such as deficiencies in $\beta$-carotene, selenium, copper, iron, iodine, and so forth. ${ }^{12-15}$ Other factors may also be considered such as removal of corpus luteum by palpation or pharmacologically, insemination of the pregnant uterus, multiple fetuses, maternal anemia, uterine or umbilical torsion, rupture of amniotic vesicle, and so forth.

Unfortunately, identifying the exact diagnosis of abortion has a relatively low success rate $(<40 \%)$. Of course, with improved clinical history, physical examination of affected animals and premises, blood tests, and postmortem evaluation, successful identification of the abortifacient is much better. There obviously is a need to advance methods and techniques to improve the exact diagnosis and to thoroughly identify causes of abortion. Readers are referred to Miller $^{12}$ for details of the diagnosis of abortion in livestock.

\section{TOXICANTS AFFECTING FERTILITY}

Toxicants causing infertility may result in a temporary reduction in reproductive function or may result in permanent dysfunction. Temporary infertility usually returns to normal when the source is removed and a period of time passes. Subtle changes in herd fertility are difficult to diagnose. Until production records are carefully evaluated and compared with past records, reductions in conception rates of $10 \%$ or less may go unrecognized. Even with good records, it is difficult to retrospectively implicate a toxicant when many other factors such as nutrition, stress, genetics, disease, and management may all contribute to such a reduction.

Toxins affecting reproduction may cause dysfunction through one or more mechanisms of action. Toxins may act directly by destroying oocytes or spermatocytes as do some alkylating agents; xenobiotics may act as hormone agonists or antagonists; or toxins may be metabolized to toxic intermediates or to putative compounds with 


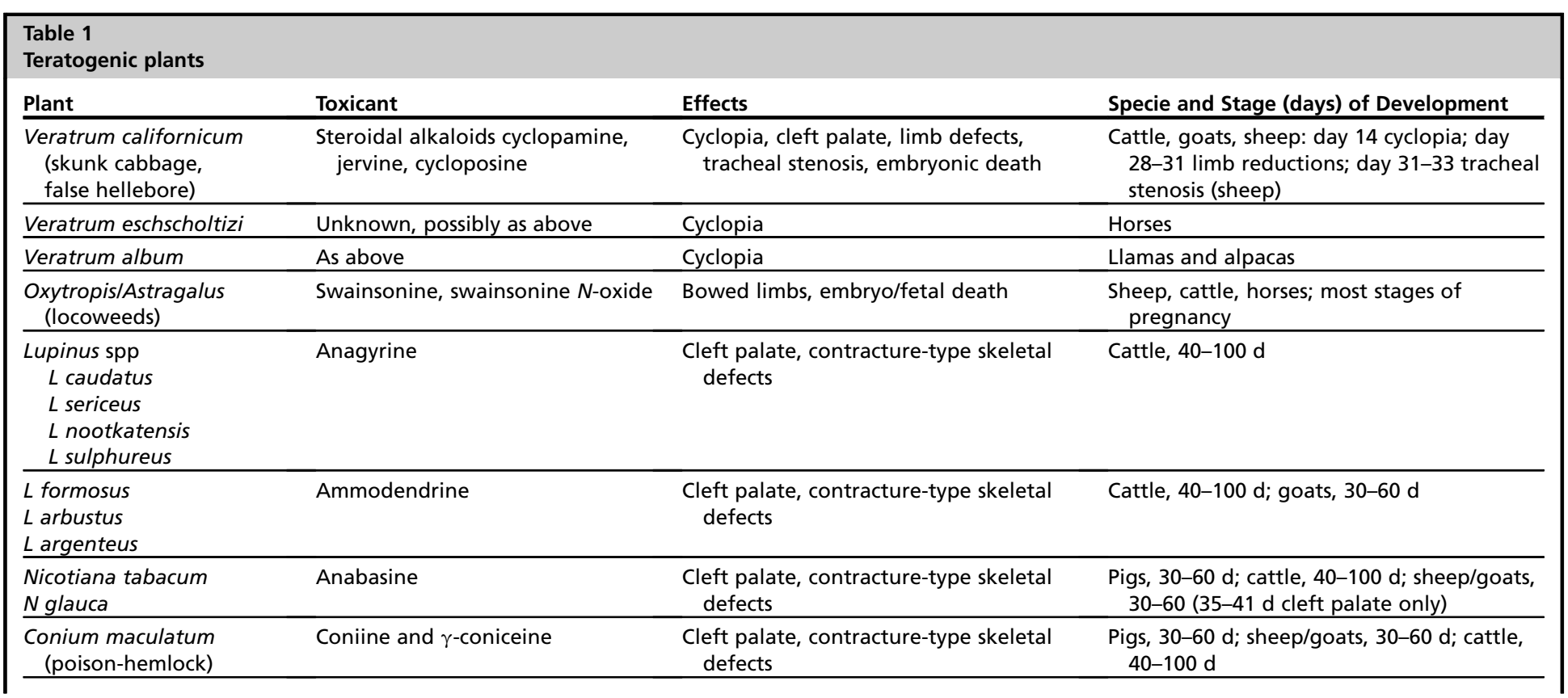




\begin{tabular}{|c|c|c|c|}
\hline $\begin{array}{l}\text { Prunus serotina } \\
\text { (wild black cherry) }\end{array}$ & Cyanogenic compounds suspected & $\begin{array}{l}\text { Cleft palate, contracture-type skeletal } \\
\text { defects }\end{array}$ & Pigs \\
\hline $\begin{array}{l}\text { Datura stramonium } \\
\text { (jimsonweed) }\end{array}$ & Unknown; possibly alkaloids & $\begin{array}{l}\text { Cleft palate, contracture-type skeletal } \\
\text { defects }\end{array}$ & Pigs \\
\hline $\begin{array}{l}\text { Sorghum vulgare } \\
\text { S sudanese }\end{array}$ & Cyanogen compounds suspected & Contracture-type skeletal defects & Horses \\
\hline $\begin{array}{l}\text { Lathyrus spp } \\
\text { L cicera } \\
\text { L odoratus }\end{array}$ & Lathyrogens & Skeletal defects & Cattle and sheep \\
\hline Ipomoea carnea & Calystegines and or Swainsonine & Fetal growth reduced & Rats, goats, rabbits: organogenesis \\
\hline Luffa acutangula & Proteins; luffin $b$ and lufaculin & Fetal growth reduced; cleft palate & Rats; postimplantation \\
\hline Mimosa tenuiflora & Unknown & $\begin{array}{l}\text { Skeletal defects, brachygnathia, cranial } \\
\text { deformities }\end{array}$ & Sheep, goats, cattle \\
\hline $\begin{array}{l}\text { Caulophyllum thalictroides } \\
\text { (Blue cohosh) }\end{array}$ & Unknown & $\begin{array}{l}\text { Cardiovascular and craniofacial cartilage } \\
\text { defects }\end{array}$ & Japanese Medaka embryos \\
\hline Aspidosperma pyrifolium & Unknown & Delayed fetal development & Rats, goats \\
\hline Senna occidentalis & Anthraquinones & Delayed behavioral development & Goats \\
\hline
\end{tabular}

Data from Refs. ${ }^{3,8,19-36}$ 


\begin{tabular}{|c|c|c|c|}
\hline \multicolumn{4}{|l|}{$\begin{array}{l}\text { Table } 2 \\
\text { Teratogenic xenobiotics }\end{array}$} \\
\hline Toxicants & Source & Effect & Species \\
\hline Parbendazole & Anthelmintic & $\begin{array}{l}\text { Vertebral column and } \\
\text { other skeletal defects }\end{array}$ & $\begin{array}{l}\text { Sheep, goats, cattle, } \\
\text { pigs }\end{array}$ \\
\hline Methallibure & Pituitary inhibitor & $\begin{array}{l}\text { Contracture-type } \\
\text { defects }\end{array}$ & $\begin{array}{l}\text { Pigs, } 30-50 \mathrm{~d} \\
\text { gestation }\end{array}$ \\
\hline Riboflavin deficiency & Vitamin & $\begin{array}{l}\text { Cleft palate, limb } \\
\text { reductions }\end{array}$ & Mammals, birds \\
\hline Sulfonamides & $\begin{array}{l}\text { Sulfur bacteriostatic } \\
\text { agents }\end{array}$ & Beak and feet defects & Chickens \\
\hline Tetrahydrophthalimide & Captan fungicides & $\begin{array}{l}\text { Skull, limb, and visceral } \\
\text { defects }\end{array}$ & Chickens \\
\hline Tryptophane & Amino acid & $\begin{array}{l}\text { Limb and visceral } \\
\text { defects }\end{array}$ & Chickens \\
\hline Trichlorfon & $\begin{array}{l}\text { Organophosphoric } \\
\text { insecticide }\end{array}$ & Cerebral hypoplasia & Pigs \\
\hline Aminoacetonitrile & $\begin{array}{l}\text { Synthetic } \\
\text { osteolathyrogen }\end{array}$ & Skeletal defects & Cattle, sheep \\
\hline Vitamin A deficiency & Vitamin & $\begin{array}{l}\text { Ocular, facial, and } \\
\text { central nervous } \\
\text { system (CNS) defects }\end{array}$ & Pigs, cattle, rabbits \\
\hline Copper deficiency & Trace element & $\begin{array}{l}\text { Skeletal and brain } \\
\text { defects }\end{array}$ & Sheep, pigs, horses \\
\hline Manganese & Trace element & $\begin{array}{l}\text { Limbs and vertebrae } \\
\text { defects }\end{array}$ & Rabbits, calves \\
\hline Molybdenum excess & Trace element & $\begin{array}{l}\text { Demyelination resulting } \\
\text { in CNS defects }\end{array}$ & Sheep \\
\hline Selenium toxicity & Trace element & Fetal hoof defects & Cattle, horses \\
\hline Aflatoxins & Aspergillus spp & Skeletal defects & Rats, goats \\
\hline $\begin{array}{l}\text { Cyanide (cyanogenic } \\
\text { glycosides) }\end{array}$ & Plants & $\begin{array}{l}\text { Skeletal contracture } \\
\text { defects }\end{array}$ & Goats \\
\hline
\end{tabular}

${ }^{a}$ Many other teratogens with reference to rodent models are found in Shepard and Lemire ${ }^{8}$ and Szabo. ${ }^{9}$

Data from Refs. $8,9,20,34,35,37-39$

structural similarities to endogenous compounds. These biologic imposters may compete at active sites or alter clearance of natural hormones.

Over the last 30 years, most of the reproductive toxicology research has focused on human reproductive vulnerability to disruption by drugs or workplace and environmental xenobiotics. ${ }^{1}$ This research and the risk assessments in humans are generally determined using rodent models, primarily mice or rats. Although research using a rodent model clearly demonstrates potential problems, the direct extrapolation of results from these models to predict toxin-induced reproductive dysfunction or sexdependent differences in xenobiotic toxicity in livestock species or humans can be inadequate or misleading.

More than 50 years ago sex-linked differences were identified in xenobiotic metabolism. This difference was first observed in rats, where the female was found to be more sensitive to the effects of barbiturates than the male. ${ }^{5}$ Subsequent studies 


\begin{tabular}{|c|c|c|c|}
\hline \multicolumn{4}{|l|}{$\begin{array}{l}\text { Table } 3 \\
\text { Abortifacient plants }\end{array}$} \\
\hline Plant & Toxicant & Effect & Species \\
\hline $\begin{array}{l}\text { Pinus, Juniperus, and other woody } \\
\text { spp needles and bark: see } \\
\text { Table } 4 \text { for specific information } \\
\text { on multiple species }\end{array}$ & $\begin{array}{l}\text { Isocupressic acid (ICA) and other } \\
\text { ICA derivatives or labdane resin } \\
\text { acids }\end{array}$ & Induced premature parturition & $\begin{array}{l}\text { Cattle and bison; anecdotal } \\
\text { information suggests llamas } \\
\text { also susceptible }\end{array}$ \\
\hline $\begin{array}{l}\text { Gutierrezia sarothrae or } \\
\text { microcephala }\end{array}$ & Unknown & $\begin{array}{l}\text { Abortion when grown on sandy } \\
\text { soil }\end{array}$ & Cattle, sheep, goats \\
\hline $\begin{array}{l}\text { Oxytropis and Astragalus } \\
\text { (locoweeds) }\end{array}$ & $\begin{array}{l}\text { Swainsonine (indolizidine } \\
\text { alkaloid) }\end{array}$ & Abortion, embryonic death & All livestock \\
\hline Swainsona spp (Australia) & Swainsonine & Similar to locoweeds & Cattle, sheep \\
\hline Vicia villosa (hairy vetch) & Unknown & Abortion & Cattle \\
\hline Leucaena leucocephala & Mimosine & Infertility, abortion & Pigs \\
\hline Aspidosperma pyrifolium & Unknown & Abortion, resorptions & Small ruminants, rats \\
\hline Tetrapterys spp & Unknown & Abortion & Goats \\
\hline Artemisia monosperma & Ethanol extracts & Abortion, resorptions & Rats \\
\hline Bambusa vulgaris & Aqueous extracts & Abortion, resorptions & Rabbits \\
\hline Ateleia glazioviana & Green or dried leaves & Abortion, stillbirth & Sheep \\
\hline
\end{tabular}

Other suspected abortifacient plants: Veratrum californicum (false hellebore), Cupressus macrocarpa (Monterey cypress), Indigofera spicata (creeping indigo), Raphanus raphanistrum (wild radish), Lantana camara, Iva augustifolia (narrow-leaf sumpweed), hybrid Sudan (Sorghum spp).

Data from Refs. $10,20,23,27,32,37,40-46$ 


\begin{tabular}{|c|c|c|c|}
\hline Species & Common Name & Location & $\begin{array}{l}\text { Isocupressic Acid Conc. } \\
\text { (\% Dry Weight) }^{\mathrm{a}}\end{array}$ \\
\hline Abies concolor & White fir & $\begin{array}{l}\text { Arizona } \\
\text { California } \\
\text { Colorado } \\
\text { Utah } \\
\end{array}$ & $\begin{array}{l}\text { n.d.b } \\
\text { n.d. } \\
0.04 \\
\text { n.d. } \\
\end{array}$ \\
\hline Abies grandis & Grand fir & $\begin{array}{l}\text { Idaho } \\
\text { Oregon }\end{array}$ & $\begin{array}{l}\text { n.d. } \\
\text { n.d. }\end{array}$ \\
\hline Abies lasiocarpa & Subalpine fir & $\begin{array}{l}\text { Oregon } \\
\text { Colorado } \\
\text { Idaho } \\
\text { Utah } \\
\end{array}$ & $\begin{array}{l}\text { n.d. } \\
\text { n.d. } \\
0.04 \\
\text { n.d. }\end{array}$ \\
\hline Abies magnifica & Red fir & California & 0.05 \\
\hline Cupressus macrocarpa & Monterey cypress & $\begin{array}{l}\text { California } \\
\text { New Zealand }\end{array}$ & $\begin{array}{l}\text { n.d. }-0.06 \\
0.89-1.24 \\
\end{array}$ \\
\hline Cupressus ovensii & - & New Zealand & 0.81 \\
\hline Juniperus californica & California juniper & $\begin{array}{l}\text { California } \\
-\end{array}$ & $\begin{array}{l}0.93 \text { needles } \\
0.05 \text { bark }\end{array}$ \\
\hline Juniperus communis & $\begin{array}{l}\text { Mountain common } \\
\text { juniper }\end{array}$ & $\begin{array}{l}\text { Colorado } \\
\text { Utah }\end{array}$ & $\begin{array}{l}2.05-2.88 \\
1.50-5.0 \\
\end{array}$ \\
\hline Juniperus monosperma & One-seed juniper & $\begin{array}{l}\text { Arizona } \\
\text { New Mexico }\end{array}$ & $\begin{array}{l}0.14 \\
\text { n.d. }\end{array}$ \\
\hline Juniperus occidentalis & Western juniper & $\begin{array}{l}\text { Oregon } \\
-\end{array}$ & $\begin{array}{l}0.10 \\
\text { Imbricatoloic acid }=1.0\end{array}$ \\
\hline Juniperus osteosperma & Utah juniper & $\begin{array}{l}\text { Utah } \\
\text { Nevada } \\
\text { Arizona } \\
\text { Colorado } \\
\text { Utah } \\
\end{array}$ & $\begin{array}{l}\text { n.d. } \\
0.07 \\
\text { n.d. } \\
\text { n.d. } \\
\text { Agathic acid = } 1.50\end{array}$ \\
\hline Juniperus scopulorum & $\begin{array}{l}\text { Rocky Mountain } \\
\text { juniper }\end{array}$ & $\begin{array}{l}\text { Utah } \\
\text { New Mexico } \\
\text { Arizona }\end{array}$ & $\begin{array}{l}0.84 \\
0.33 \\
0.42 \\
\end{array}$ \\
\hline Juniperus virginiana & Eastern red cedar & Nebraska & $\begin{array}{l}\text { needles, low } \\
\text { bark, }<0.10 \text {-high }\end{array}$ \\
\hline Larix occidentalis & Western larch & Oregon & n.d. \\
\hline Libocedrus decurrens & Incense cedar & Oregon & 0.07 \\
\hline Picea engelmannii & Engelmann spruce & $\begin{array}{l}\text { California } \\
\text { Colorado } \\
\text { Idaho } \\
\text { Montana } \\
\text { Oregon } \\
\text { Utah } \\
\end{array}$ & $\begin{array}{l}0.27 \\
\text { n.d } \\
0.04 \\
0.31 \\
\text { n.d. } \\
\text { n.d. }\end{array}$ \\
\hline Picea pungens & Colorado blue spruce & $\begin{array}{l}\text { Utah } \\
\text { Colorado }\end{array}$ & $\begin{array}{l}0.17 \\
\text { n.d. }\end{array}$ \\
\hline Pinus aristata & Bristle cone pine & Colorado & $0.01-0.05$ \\
\hline Pinus arizonica & Arizona pine & $\begin{array}{l}\text { California } \\
\text { Arizona }\end{array}$ & $\begin{array}{l}\text { n.d. } \\
\text { n.d. }\end{array}$ \\
\hline & & & (continued on next page) \\
\hline
\end{tabular}




\begin{tabular}{|c|c|c|c|}
\hline \multicolumn{4}{|l|}{$\begin{array}{l}\text { Table } 4 \\
\text { (continued) }\end{array}$} \\
\hline Species & Common Name & Location & $\begin{array}{l}\text { Isocupressic Acid Conc. } \\
\text { (\% Dry Weight) }^{\text {a }}\end{array}$ \\
\hline Pinus contorta & Lodgepole pine & $\begin{array}{l}\text { Oregon } \\
\text { Idaho } \\
\text { Colorado } \\
\text { Utah } \\
\text { Canada (BC) } \\
\end{array}$ & $\begin{array}{l}0.28 \\
0.11 \\
0.29-0.47 \\
0.66 \\
0.45 \\
\end{array}$ \\
\hline Pinus densiflora & Japanese redpine & Korea & n.d. \\
\hline Pinus echinata & Short-leaf pine & Arkansas & n.d. \\
\hline Pinus edulis & Pinyon pine & $\begin{array}{l}\text { Arizona } \\
\text { Colorado } \\
\text { New Mexico } \\
\text { Utah }\end{array}$ & $\begin{array}{l}\text { n.d. } \\
0.12 \\
0.10 \\
0.45 \\
\end{array}$ \\
\hline$\underline{\text { Pinus elliottii }}$ & Slash pine & Arkansas & n.d. \\
\hline Pinus flexilis & Limber pine & $\begin{array}{l}\text { Colorado } \\
\text { Utah }\end{array}$ & $\begin{array}{l}\text { n.d. }-0.06 \\
\text { n.d. }\end{array}$ \\
\hline Pinus halepensis & Aleppo pine & California & n.d. \\
\hline Pinus jeffreyi & Jeffrey pine & California & $0.04-0.54$ \\
\hline Pinus koraiensis & Korean pine & $\begin{array}{l}\text { Utah } \\
\text { Korea } \\
\end{array}$ & $\begin{array}{l}\text { Positive } \\
0.02\end{array}$ \\
\hline Pinus monophylla & Single-leaf pinyon & Nevada & 0.32 \\
\hline Pinus montezumae & Montezuma pine & California & n.d. \\
\hline Pinus palustris & Long-leaf pine & Arkansas & n.d. \\
\hline Pinus patula & Patula pine & South Africa & $<0.10$ \\
\hline Pinus ponderosa & Ponderosa pine & $\begin{array}{l}\text { Oregon } \\
\text { Arizona } \\
\text { California } \\
\text { Utah } \\
\text { Colorado } \\
\text { South Dakota } \\
\text { Wyoming } \\
\text { Germany } \\
\end{array}$ & $\begin{array}{l}0.74-1.30 \\
0.49 \\
0.08-1.35 \\
0.51 \\
0.49-0.58 \\
0.10-1.30 \\
0.58-1.11 \\
0.62 \\
\end{array}$ \\
\hline Pinus radiata & Radiata pine & New Zealand & n.d. -0.26 \\
\hline Pinus taeda & Loblolly pine & $\begin{array}{l}\text { Arizona } \\
\text { Arkansas }\end{array}$ & $\begin{array}{l}\text { n.d. } \\
\text { n.d. }\end{array}$ \\
\hline Pseudotsuga menziesii & Douglas fir & $\begin{array}{l}\text { Utah } \\
\text { Colorado } \\
\text { California } \\
\text { Idaho } \\
\text { Arizona } \\
\text { Oregon } \\
\end{array}$ & $\begin{array}{l}0.04 \\
0.05 \\
\text { n.d. } \\
\text { n.d. } \\
\text { n.d. } \\
\text { n.d }\end{array}$ \\
\hline Thuja plicata & Western red cedar & $\begin{array}{l}\text { Arizona } \\
\text { New Mexico } \\
\text { Utah } \\
\text { Germany }\end{array}$ & $\begin{array}{l}0.42 \\
0.33 \\
0.84 \\
\text { n.d. }\end{array}$ \\
\hline Tsuga mertensiana & Mountain hemlock & Oregon & n.d. \\
\hline
\end{tabular}

a Values are for measured concentrations of isocupressic acid, or where otherwise noted, may include the measurement of a similar related diterpene in samples where the indicated compound was identified and was also the major labdane acid present in the sample.

${ }^{b}$ n.d., not detected $(<0.01 \%)$.

Data from Gardner DR, Molyneux RJ, James LF, et al. Ponderosa pine needle-induced abortion in beef cattle: identification of isocupressic acid as the principal active compound. J Agric Food Chem 1994;42:756; and Yakubu MT, Bukoye BB, Oladiji AT, et al. Toxicological implication of aqueous extract of Bambusa vulgaris leaves in pregnant Dutch rabbits. Hum Exp Toxicol 2009;28:591. 


\begin{tabular}{|c|c|c|c|}
\hline \multicolumn{4}{|c|}{$\begin{array}{l}\text { Table } 5 \\
\text { Mycotoxins associated with abortions }\end{array}$} \\
\hline Fungi & Toxicant & Effect & Species \\
\hline $\begin{array}{l}\text { Claviceps spp } \\
\text { (infected grains } \\
\text { and grasses) }\end{array}$ & Ergot alkaloids & $\begin{array}{l}\text { Vasoconstriction, } \\
\text { abortion }\end{array}$ & $\begin{array}{l}\text { Pigs, cattle, horse, } \\
\text { sheep }\end{array}$ \\
\hline $\begin{array}{l}\text { Acremonium } \\
\text { coenophialum } \\
\text { (endophyte- } \\
\text { infected fescue) }\end{array}$ & $\begin{array}{l}\text { Perloline, peramine, } \\
\text { formylloline }\end{array}$ & Stillbirths, abortion & $\begin{array}{l}\text { Cattle, horses, } \\
\text { sheep, } \\
\text { pigs, rabbits }\end{array}$ \\
\hline $\begin{array}{l}\text { Balansia spp } \\
\quad \text { (infected grasses) }\end{array}$ & Ergot alkaloids & $\begin{array}{l}\text { Similar to Claviceps } \\
\text { spp }\end{array}$ & Cattle \\
\hline $\begin{array}{l}\text { Fusarium spp } \\
\text { (trichothecenes) }\end{array}$ & $\begin{array}{l}\text { Diacetoxyscirpenol } \\
\text { (DAS) }\end{array}$ & $\begin{array}{l}\text { Feed refusal, } \\
\text { nausea, abortion }\end{array}$ & Cattle, pigs \\
\hline $\begin{array}{l}\text { Aspergillus spp and } \\
\text { Penicillium spp }\end{array}$ & $\begin{array}{l}\text { Ochratoxins } \\
\text { (isocoumarins and } \\
\text { phenylalanine } \\
\text { derivatives) }\end{array}$ & $\begin{array}{l}\text { Nephropathy, } \\
\text { enteritis in swine; } \\
\text { abortion in cattle }\end{array}$ & Pigs, cattle \\
\hline
\end{tabular}

Other mycotoxins suspected to cause abortion include: Penicillium roqueforti contaminated grains and corn silage reported in cattle; Stachybotrys alterrans contaminated hay or straw reported to cause anorexia, necrotic dermatitis, ulcerative lesions in horses, cattle, sheep and swine, and abortions in swine and cattle in terminal stages; and phomopsins from infected sweet lupines.

Data from Refs. ${ }^{15,20,37,47,48}$

demonstrated that in general, male rats have higher rates of xenobiotic metabolism than do females. This finding was further supported when experiments determined that female rats have $10 \%$ to $30 \%$ less total cytochrome P450 enzymes than male rats. ${ }^{13}$ These sex-dependent differences are further demonstrated in the expression of cytochrome P450 isoforms that catalyze the hydroxylation of steroids. ${ }^{16}$ While most literature on sex-dependent differences uses the rat model, there is some limited research suggesting similar sex-dependent differences in metabolism in mice, rabbits, dogs, monkeys, and humans. ${ }^{1}$ In livestock there is very little if any research demonstrating similar differences.

Certain reproductive disorders in male children have been increasing in the last decade. ${ }^{17}$ Reproductive tract abnormalities such as cryptorchidism, hypospadias, and testicular cancer are increasing in certain human populations. Declining sperm counts have also been reported in certain areas of the world. Similar reproductive disorders have been reported in wildlife species, and have been suggested to be caused by highly contaminated ecosystems. Although few of these types of reproductive disorders in livestock species have been associated with environmental toxicants, more and more anecdotal links are sure to come forth in this regard.

Where xenobiotic-induced reproductive dysfunction is suspected in livestock species, if at all possible the suspected toxicant should be evaluated in the target species. More research is needed to identify toxicants that have adverse impacts on livestock reproduction. Toxicants known to affect male and female fertility are listed in Tables 7 and 8, and those affecting fowl are listed in Table 9. 


\begin{tabular}{|c|c|c|c|}
\hline \multicolumn{4}{|c|}{$\begin{array}{l}\text { Table } 6 \\
\text { Other xenobiotics associated with abortion }\end{array}$} \\
\hline Toxicant & Source & Effect & Species \\
\hline Nitrates/nitrites & Plants, water, fertilizers & Poor growth, infertility, abortion, death & Ruminants most susceptible \\
\hline High protein diets, excess urea & $\begin{array}{l}\text { Immature high protein pastures, } \\
\text { high urea added to diet }\end{array}$ & Abortion, embryonic death & Cattle \\
\hline Carbon monoxide & $\begin{array}{l}\text { Incomplete combustion, poor } \\
\text { ventilation }\end{array}$ & $\begin{array}{l}\text { Inhibited respiratory function, abortion, } \\
\text { stillbirths }\end{array}$ & Pigs; all species potentially affected \\
\hline Estrogens/phytoestrogens & Plants, silage, pharmaceuticals & Abortion, infertility, anestrus & Sheep sensitive; other species affected \\
\hline Glucocorticoids & Pharmaceuticals & Abortion, retained placenta & All species \\
\hline $\begin{array}{l}\text { Halogenated dioxins and } \\
\text { related compounds }\end{array}$ & $\begin{array}{l}\text { Wood preservatives, lubricants, } \\
\text { solvents }\end{array}$ & Hypovitaminosis, abortion & Cattle \\
\hline Lead & $\begin{array}{l}\text { Discarded materials, paint, greases, } \\
\text { batteries }\end{array}$ & $\begin{array}{l}\text { Ataxia, head pressing, encephalopathy, } \\
\text { abortion suspected }\end{array}$ & All species \\
\hline Phenothiazine & Anthelmintic & $\begin{array}{l}\text { Primary photosensitization, abortion } \\
\text { suspected }\end{array}$ & Cattle, sheep, horses, pigs \\
\hline Prostaglandins & Pharmaceuticals & Abortions, retained placenta & All species \\
\hline Oxytocin & Pharmaceuticals & Induced parturition & Horses \\
\hline DDT, dieldrin, heptachlor & Pesticides & Residues detected in aborted fetuses & Cattle \\
\hline Warfarin (coumarin) & Rodent bait & Abortion & Cattle \\
\hline
\end{tabular}

Data from Refs. ${ }^{14,15,20,37}$ 
Table 7

Toxicants causing infertility

\section{Toxicant}

Phytoestrogens

Coumestrol

Daidzein

Biochanin A

Formononetin

Zearalenone

Zearalenol A and B

Steroidal estrogens

Diethylstilbestrol (DES)

Wheat germ

o,p'-DDT

Ergot

Swainsonine

Mimosine

Selenium

Source

Trifolium subterraneum (subterranean clover), Medicago sativa, Medicago truncata (alfalfa)

Fusarium roseum

Rayless goldenrod

Pharmaceutical (discontinued)

Wheat grains

DDT metabolite

Ergot sclerotia (seed grains)

Locoweeds, Swainsona spp

Leucaena leucocephala

Grasses and forbs on seleniferous soils

\section{Effect}

Infertility, decreased conception, irregular estrous cycles
Species

Sheep, cattle, horses

Pigs most susceptible; other species affected

Vulvovaginitis

Anestrus, uterine hypertrophy, anovulatory estrus

Lactation in unbred ewes and wethers; dystocia, Sheep infertility

Transplacental carcinogen, abnormal development in fetal reproductive tract

Estrogenic effects

Estrogen effects (egg shell thinning)

Infertility, abortion, agalactia, decreased prolactin

Early embryonic loss, decreased estrus behavior, reduced ovulation rate, abortion

Infertility, abortion

Inhibits estrous cycle in excess and may increase spontaneous abortion if deficient

\begin{tabular}{|c|c|c|c|}
\hline$\beta$-Carotene & Excess vitamin A & Reduced conception rates & Dairy cattle \\
\hline Glucosinolates & Brassica spp & Infertility & All species \\
\hline Ethanol extract & Abrus precatorius L. & Infertility/DNA damage to spermatozoa & Mice \\
\hline Alcohol extract & Neem flower & Blocks ovulation & Rats \\
\hline
\end{tabular}

Treatment generally involves removal of source and recovery is usually spontaneous.

Data from Refs. ${ }^{17,20,37,48-50}$ 


\begin{tabular}{|c|c|c|c|}
\hline \multicolumn{4}{|c|}{$\begin{array}{l}\text { Table } 8 \\
\text { Toxicants affecting male reproduction }\end{array}$} \\
\hline Toxicant & Source & Effect & Species \\
\hline Swainsonine & Locoweed & $\begin{array}{l}\text { Decreased libido, reduced sperm } \\
\text { production, increased abnormal } \\
\text { sperm }\end{array}$ & All species \\
\hline Gossypol & Cotton seed meal & $\begin{array}{l}\text { Blocks spermatogenesis, reduces } \\
\text { sperm motility, male infertility, } \\
\text { testicular degeneration }\end{array}$ & All species \\
\hline Phytoestrogens & Clover and alfalfa (see Table 6) & $\begin{array}{l}\text { Mammary development in wethers, } \\
\text { reduced libido in rams }\end{array}$ & $\begin{array}{l}\text { Sheep most susceptible; cattle } \\
\text { affected }\end{array}$ \\
\hline Boric acid & $\begin{array}{l}\text { Commercial applications (roach } \\
\text { control, therapeutic and industrial } \\
\text { products) }\end{array}$ & $\begin{array}{l}\text { Altered spermatogenesis, decreased } \\
\text { sperm motility, increased abnormal } \\
\text { sperm }\end{array}$ & Laboratory species \\
\hline $\begin{array}{l}\text { Anabolic steroids and } \\
\text { androgenic hormones }\end{array}$ & Pharmaceuticals & $\begin{array}{l}\text { Prolonged use results in } \\
\text { masculinization, testicular } \\
\text { degeneration }\end{array}$ & Horses; most species \\
\hline Halogenated dioxins & Solvents, lubricants & Masculinization & Horses \\
\hline Chlorinated naphthalene & $\begin{array}{l}\text { Solvents, wood preservatives, } \\
\text { lubricants }\end{array}$ & Testicular degeneration & All species \\
\hline Acute cadmium toxicosis & Industrial contaminant, anthelmintic & Testicular degeneration & All species \\
\hline
\end{tabular}

Treatment: Observed changes in spermatogenesis are usually delayed 30 to 60 days, whereas changes in libido may be immediate; treatment usually involves removal of the source and recovery will occur spontaneously. With anabolic steroids, halogenated dioxins, chlorinated naphthalenes, and cadmium toxicosis, the effects may be permanent.

Data from Refs. ${ }^{8,20,32,37}$ 


\begin{tabular}{|c|c|c|c|}
\hline $\begin{array}{l}\text { Table } 9 \\
\text { Toxicants affecting fowl reproduction }\end{array}$ & & & \\
\hline Toxicant & Source & Effect & Species \\
\hline Gossypol & Cotton seed meal & $\begin{array}{l}\text { Green yolks, pink albumin, decreased } \\
\text { hatchability of eggs }\end{array}$ & Poultry \\
\hline Aflatoxin, citrinin, patulin & Aspergillus spp & Thickened egg shells & Chickens (residues) \\
\hline $\begin{array}{l}\text { DAS (diacetoxyscirpenol) and others in } \\
\text { Fusarium; DON (deoxynivalenol) and } \\
\text { zearalenone }\end{array}$ & Fusarium roseum & $\begin{array}{l}\text { Decreased hatchability, reduced egg } \\
\text { production and egg shell weight }\end{array}$ & Chickens \\
\hline Ammonia & Pit gases & Decreased egg production & Chickens \\
\hline Lindane & Organochlorine pesticide & Smaller clutch size, reduced yolk protein & Ducks \\
\hline DDT (DDE) & $\begin{array}{l}\text { Organochlorine pesticide (illegal); } \\
\text { residue still in environment }\end{array}$ & $\begin{array}{l}\text { Reduced egg shell thickness, poor hatch, } \\
\text { lighter eggs, delayed ovulation, } \\
\text { reduced clutch size }\end{array}$ & All fowl \\
\hline Lead & Lead-containing products & Reduced egg production & Chickens, quail \\
\hline $\begin{array}{l}\text { Thiocarbamates (thiram, ziram, ferbam, } \\
\text { maneb, zineb) }\end{array}$ & Fungicides & $\begin{array}{l}\text { Retarded testicular growth, abnormal } \\
\text { seminiferous tubules, infertility }\end{array}$ & Chickens and other fowl \\
\hline Selenium & $\begin{array}{l}\text { Runoff from Se soils and additives to } \\
\text { feeds }\end{array}$ & Decreased hatchability & All fowl \\
\hline Glucosinolates & Brassica spp, crambe meal rapeseed, etc & $\begin{array}{l}\text { Decreased egg production, off-flavored } \\
\text { eggs, embryo thyroid enlargement }\end{array}$ & Chickens, turkeys \\
\hline Linatine, linamarin & Raw soybean meal & Decreased growth & Chickens \\
\hline Phytoestrogens & $\begin{array}{l}\text { Red clover, subterranean clover } \\
\text { (phytoestrogens during dry conditions) }\end{array}$ & Decreased reproduction rate & California quail \\
\hline Mimosine & Leucaena leucocephala & Infertility at $>10 \%$ Leucaena in diet & Chickens \\
\hline
\end{tabular}

Data from Refs. ${ }^{18,20,51-56}$ 


\section{SUMMARY}

With the technological advances made in molecular biology, biochemistry, chemical detection, and toxicology, reproductive toxicology has made significant progress in the identification of toxins and mechanisms of action. However, much of the reproductive toxicology research has been done in rodent models and may or may not be totally applicable to food-producing animals or humans. Novel molecular and biochemical probes will enable investigators to move to higher levels of sophistication in their search for mechanisms of action. ${ }^{18}$ The charge to protect human health, animal health, and the environment from reproductive toxicants is a challenging one. An effective response will require the talents of multidisciplinary teams of scientists applying novel ideas and techniques. In this article the authors attempt to provide brief information in tabular form for rapid reference with regard to food-producing species. Although this list is undoubtedly incomplete, it demonstrates the extent and complexity of diagnosing the causes of reproductive dysfunction.

\section{REFERENCES}

1. Kedderis GL, Mugford CA. Sex-dependent metabolism of xenobiotics. Chemical Industry Institute of Toxicology 1998;18(7-8):1.

2. Wilson JG. Current status of teratology. In: Wilson JG, Fraser FC, editors. Handbook of teratology. New York: Plenum Press; 1977. p. 476.

3. Panter KE, James LF, Gardner DR. Lupine, poison-hemlock and Nicotiana spp: toxicity and teratogenicity in livestock. J Nat Toxins 1999;8:117.

4. Nelson K, Holmes LB. Malformations due to presumed spontaneous mutations in new born infants. N Engl J Med 1989;320:19.

5. Nelson DR, Koymans L, Kamataki T, et al. P450 superfamily: update on new sequences, gene mapping, accession numbers and nomenclature. Pharmacogenetics 1996;6:1-42.

6. Gaffield W. The Veratrum alkaloids: natural tools for studying embryonic development. In: Atta-ur-Rahman, editor, Studies in natural products chemistry, vol. 23. Amsterdam: Elsevier; 2000. p. 563-89.

7. Gaffield W, Keeler RF. Steroidal alkaloid teratogens: molecular probes for investigation of craniofacial malformations. J Toxicol Toxin Rev 1996;15:303.

8. Shepard TH, Lemire RJ. Catalog of teratogenic agents. 13th edition. Baltimore (MD): John Hopkins University Press; 2011.

9. Szabo KT. Congenital malformations in laboratory and farm animals. San Diego (CA): Academic Press; 1989. p. 8-93.

10. Gardner DR, Molyneux RJ, James LF, et al. Ponderosa pine needle-induced abortion in beef cattle: identification of isocupressic acid as the principal active compound. J Agric Food Chem 1994;42:756.

11. Welch KD, Davis TZ, Panter KE, et al. The effect of poisonous range plants on abortions in livestock. Rangelands 2009;31:28.

12. Miller RB. Bovine abortion. In: Morrow AA, editor. Current therapy in theriogenology: diagnosis, treatment and prevention of reproductive diseases in small and large animals. Philadelphia: WB Saunders; 1986. p. 291.

13. Holch HG, Munir AK, Mills LM, et al. Studies upon the sex-difference in rats in tolerance to certain barbiturates and to nicotine. J Pharmacol Exp Ther 1937; 60:323.

14. Norton JH, Campbell RS. Non-infectious causes of bovine abortion. Vet Bull 1989; 60:1137. 
15. Osweiler GD, Carson TL, Buck WB, et al. Diagnostic toxicology. In: Clinical and diagnostic veterinary toxicology. 3rd edition. Dubuque (IA): Kendall/Hunt; 1985. p. 44-51.

16. Waxman DJ, Dannan GA, Gurngerich FP. Regulation of rat hepatic cytochrome P-450: age-dependent expression, hormonal imprinting, and xenobiotic inducibility of sex-specific isoenzymes. Biochemistry 1985;24:4409.

17. Mylchreest E, Foster PM. Antiandrogenic effects of Di(n-Butyl) phthalate on male reproductive development: a nonreceptor-mediated mechanism. Chemical Industry Institute of Toxicology 1998;1:181.

18. Edema FW, Garlick JD. Lead-induced egg production decrease in leghorn and Japanese quail hens. Poult Sci 1983;62:1757.

19. Barbosa-Ferreira M, Pfister JA, Gotardo AT, et al. Effects of Senna occidentalis seeds ingested during gestation on kid behavior. In: Riet-Correa F, Pfister J, Schild AL, et al, editors. Poisoning by plants, mycotoxins, and related toxins. Wallingford (CT): Oxfordshire: CAB International; 2011. p. 264-9.

20. Cheeke PR. Natural toxicants in feeds, forages and poisonous plants. Danville (IL): Interstate Publishers; 1998. p. 479.

21. Dugoua JJ, Perri D, Seely D, et al. Safety and efficacy of blue cohosh (Caulophyllum thalictroides) during pregnancy and lactation. Can J Clin Pharmacol 2008;15:66.

22. Fernandes LC, Cordeiro LA, Soto-Blanco B. Evaluation of the abortifacient effect of Luffa acutangula Roxb. in rats. In: Riet-Correa F, Pfister J, Schild AL, et al, editors. Poisoning by plants, mycotoxins, and related toxins. Wallingford (CT): Oxfordshire: CAB International; 2011. p. 270-3.

23. Figueiredo AP, Dantas FP, Medeiros RM, et al. Determination of teratogenic effects of Aspidosperma pyrifolium ethanolic extract in rats. In: Riet-Correa F, Pfister J, Schild AL, et al, editors. Poisoning by plants, mycotoxins, and related toxins. Wallingford (CT): Oxfordshire: CAB International; 2011. p. 280-4.

24. Gaffield W, Indardona JP, Kapur RP, et al. Mechanistic investigation of Veratrum alkaloid-induced mammalian teratogenesis. ACS Symposium Series \#745. In: Tu AT, Gaffield W, editors. Natural and selected toxins: biological implications. Washington, DC: American Chemical Society; 2000. p. 173-87.

25. Hueza IM, Guerra JL, Haraguchi M, et al. Assessment of the perinatal effects of maternal ingestion of Ipomoea carnea in rats. Exp Toxicol Pathol 2007;58:439.

26. Keeler RF. Congenital malformations caused by poisonous plants. Comp Pathol Bull 1991;23:1.

27. Kingsbury JM. Poisonous plants of the United States and Canada. Englewood Cliffs (NJ): Prentice Hall; 1964. p. 460-1.

28. Lima MCJS, Soto-Blanco B. Experimental studies of poisoning by Aspidosperma pyrifolium. In: Riet-Correa F, Pfister J, Schild AL, et al, editors. Poisoning by plants, mycotoxins, and related toxins. Wallingford (CT): Oxfordshire: CAB International; 2011. p. 274-9.

29. Lippi LL, Santos FM, Moreira CQ, et al. Toxic effects of Ipomoea carnea on placental tissue of rats. In: Riet-Correa F, Pfister J, Schild AL, et al, editors. Poisoning by plants, mycotoxins, and related toxins. Wallingford (CT): Oxfordshire: CAB International; 2011. p. 251-5.

30. Medeiros RM, Figueiredo AP, Benicio TM, et al. Teratogenicity of Mimosa tenuiflora seeds to pregnant rats. Toxicon 2007;51:316.

31. Panter KE, Bunch TD, Keeler RF, et al. Multiple congenital contracture (MCC) and cleft palate induced in goats by ingestion of piperidine alkaloid-containing plants: reduction in fetal movement as the probable cause. J Toxicol Clin Toxicol 1990; 28:69. 
32. Panter KE, James LF, Stegelmeier BL. Locoweeds: effects on reproduction in livestock. J Nat Toxins 1999;8:53.

33. Panter KE, Keeler RF. Induction of cleft palate in goats by Nicotiana glauca during a narrow gestational period and the relation to reduction in fetal movement. $J$ Nat Toxins 1992;1:25.

34. Rousseaux CG, Blakley PM. Fetus. In: Haschek W, Rousseaux CB, editors. Handbook of toxicologic pathology. San Diego (CA): Academic Press; 1991. p. 937.

35. Rousseaux CG, Ribble CS. Developmental anomalies in farm animals II. Defining etiology. Can Vet J 1988;28:30.

36. Wu M, Hu Y, Ali Z, et al. Teratogenic effects of blue cohosh (Caulophyllum thalictroides) in Japanese medaka (Oryzias latipes) are probably mediated through GATA2/EDN1 signaling pathway. Chem Res Toxicol 2010;23:1405.

37. Putnam MR. Toxicologic problems in food animals affecting reproduction. Vet Clin North Am Food Anim Pract 1989;5:325.

38. Soto-Blanco B, Gorniak SL. Prenatal toxicity of cyanide in goats: a model for teratological studies in ruminants. Theriogenology 2004;62:1012.

39. de Carvalho PR, Pita MC, Loureiro JE, et al. Manganese deficiency in bovines: connection between manganese metalloenzymes dependent in gestation and congenital defects in newborn calves. Pakistan J Nut 2010;9:488.

40. Hijaza AM, Salhab AS. Effects of Artemisia monosperma ethanolic leaves extract on implantation, mid-term abortion and parturition of pregnant rats. J Ethnopharmacol 2010;128:446.

41. Panter KE, James LF. A review of pine needle and broom snakeweed abortion in cattle. Proceedings: Symposium on Plant-Herbivore Interactions. Washington, DC: USDA Forest Service General Technical Report INT-222;1987. p. 125.

42. Panter KE, James LF, Molyneux RJ. Ponderosa pine needle-induced parturition in cattle. J Anim Sci 1992;70:1604.

43. Peixoto PV, Caldas SA, Franca TN, et al. Chronic heart failure and abortion caused by Tetrapterys spp. in cattle in Brazil. In: Riet-Correa F, Pfister J, Schild $A L$, et al, editors. Poisoning by plants, mycotoxins, and related toxins. Wallingford (CT): Oxfordshire: CAB International; 2011.

44. Raffi MB, Barros RR, Graganca JFM, et al. The pathogenesis of reproductive failure induced in sheep by the ingestion of Ateleia glazioviana. Vet Hum Toxicol 2004;46:233.

45. Riet-Correa G, Riet-Correa F, Schild Al, et al. Abortion and neonatal mortality in sheep poisoned to Tetrapterys multiglandulosa. Vet Pathol 2009;46:960.

46. Yakubu MT, Bukoye BB, Oladiji AT, et al. Toxicological implication of aqueous extract of Bambusa vulgaris leaves in pregnant Dutch rabbits. Hum Exp Toxicol 2009;28:591.

47. Lynch GP. Biological effects of mycotoxins on ruminants. In: National Research Council, editor. Interaction of mycotoxins in animal production. Washington, DC: National Academy of Sciences; 1979. p. 96-117.

48. Porter JK, Thompson FN Jr. Effects of fescue toxicity on reproduction in livestock. J Anim Sci 1992;70:1594.

49. Gbotolorun SC, Osinubi AA, Noronha CC, et al. Antifertility potential of neem flower extract on adult female Sprague-Dawley rats. Afr Health Sci 2008;8: 168.

50. Jahan S, Rasool S, Khan MA, et al. Antifertility effects of ethanolic seed extract of Abrus precatorius L. on sperm production and DNA integrity in adult male mice. J Med Plants Res 2009;3:809. 
51. Abdelhamid AM, Dorra TM. Study on effects of feeding laying hens on separate mycotoxins (aflatoxins, patulin or citrinin)-contaminated diets on egg quality and tissue constituents. Arch Tierernahr 1990;40:305 Berlin.

52. Branton SL, Deaton JW, Hagler WM Jr, et al. Decreased egg production in commercial laying hens fed zearalenone- and deoxynivalenol-contaminated grain sorghum. Avian Dis 1989;33:804.

53. Chakravarty S, Mandal A, Lahiri P. Effect of lindane on clutch size and level of egg yolk protein in domestic duck. Toxicology 1986;39:93.

54. Chowdhury SD, Davis RH. Lathyrism in laying hens and increases in egg weight. Vet Rec 1988;123:272.

55. Deaton JW, Reece FN, Lott BD. Effect of atmospheric ammonia on laying hen performance. Poult Sci 1982;61:1815.

56. Serio R, Long RA, Taylor JE. The antifertility and antiadrenergic actions of thiocarbamate fungicides in laying hens. Toxicol Appl Pharmacol 1984;72:333. 